\title{
Modeling of optoelectronic devices with one-band effective mass equation: nonequilibrium Green's function approach
}

\author{
Andrzej Kolek ${ }^{1}$
}

Received: 1 October 2015/Accepted: 8 January 2016/Published online: 21 January 2016

(C) The Author(s) 2016. This article is published with open access at Springerlink.com

\begin{abstract}
One-band one-dimensional effective mass model useful in the simulations of layered n-type devices is proposed. The model preserves nonparabolicity both in transport and in-plane directions and enables calculations of intersubband absorption. It is integrated into nonequilibrium Green's function method which is used to simulate quantum cascade laser.
\end{abstract}

Keywords One-band effective mass approximation · In-plane dispersion ·

Nonequilibrium Green's function · Quantum cascade laser · Intersubband absorption

\section{Introduction}

When modeling unipolar devices, a one-band effective mass equation (EME) is the first choice. The main benefit of this approach is the saving of computer resources which can be then used for more complex description that takes into account quantum coherence and scattering. For optoelectronic devices utilizing intersubband optical transition in $\mu \mathrm{m}$ range, the energy levels are raised well above the band bottom, where they are strongly influenced by the remote bands. One-band description can account for this effect, e.g., by the use of energy-dependent effective mass. For layered n-type devices, an efficient approximation is one dimensional (1D) energy-dependent EME

This article is part of the Topical Collection on Numerical Simulation of Optoelectronic Devices, NUSOD' 15.

Guest edited by Julien Javaloyes, Weida Hu, Slawek Sujecki and Yuh-Renn Wu.

Andrzej Kolek

akoleknd@prz.edu.pl

1 Department of Electronics Fundamentals, Rzeszow University of Technology, Al. Powstancow Warszawy 12, 35-959 Rzeszow, Poland 


$$
\frac{-\hbar^{2}}{2} \frac{d}{d z} \frac{1}{m(E, z)} \frac{d f}{d z}+\left(E_{c}(z)+\frac{\hbar^{2} k^{2}}{m(E, z)}\right) f=E f
$$

which uses bulk effective mass $(m)$ at the total energy $(E)$ for both in-plane and longitudinal $(z)$ kinetic energy terms. The efficiency of Eq. (1) stems from the fact that it preserves in-plane nonparabolicity that matches well the results of an 8-band kp model (Faist 2013). However, the solutions of Eq. (1) are not orthonormal and so cannot be used for the evaluation of momentum matrix elements which requires orthonormal wavefunctions. Usually, this difficulty is overcome by solving the two-band (TB) Hamiltonian which gives orthogonal, two-component wavefunctions $(f, g)$ composed of the function $f(z)$ completed with the fictitious valence band component $g(z)$ (Leavitt 1991; Sirtori et al. 1994). For the Hamiltonian described by Eq. (1), its TB counterpart

$$
\left[\begin{array}{cc}
E_{c}(z)+\frac{\hbar^{2} k^{2}}{2 m(E)} & \frac{\hbar^{2}}{2 m^{*} \sqrt{\gamma}} \frac{d}{d z} \\
-\frac{\hbar^{2}}{2 m^{*} \sqrt{\gamma}} \frac{d}{d z} & E_{v}(z)
\end{array}\right]\left[\begin{array}{l}
f \\
g
\end{array}\right]=E\left[\begin{array}{l}
f \\
g
\end{array}\right]
$$

is still energy-dependent, and so the solutions $(f, g)$ are still not orthogonal and useless in the calculations of dipole matrix elements.

In this paper, another effective mass approximation is proposed which, on the one hand, matches well the in-plane dispersion predicted by the 8-band $\mathrm{kp}$ model and, on the other hand, reduces to the longitudinal Hamiltonian which has an energy-independent TB counterpart. Then, intersubband absorption can be rigorously treated because the twocomponent eigenfunctions $(f, g)$ of such Hamiltonian are orthogonal.

\section{Anisotropic nonparabolicity}

Instead of Eq. (1), one may consider EME

$$
\begin{gathered}
\frac{-\hbar^{2}}{2} \frac{d}{d z} \frac{1}{m\left(E_{z}, z\right)} \frac{d f}{d z}+\left(E_{c}(z)+\frac{\hbar^{2} k^{2}}{m\left(E, z_{\mathrm{aw}}\right)}\right) f=E f, \\
E_{z}=E-\frac{\hbar^{2} k^{2}}{2 m\left(E, z_{\mathrm{aw}}\right)},
\end{gathered}
$$

where different effective masses are used in transport and in-plane directions. The in-plane dispersion

$$
E=\frac{E_{g}}{2}\left\{-1+\frac{E_{i}}{E_{g}}+\sqrt{\left(1+\frac{E_{i}}{E_{g}}\right)^{2}+\frac{2 \hbar^{2} k^{2}}{m^{*} E_{g}}}\right\},
$$

for a subband originated at energy level $E_{z}=E_{i}$ can be obtained inserting $m\left(E, z_{\text {aw }}\right)=$ $m^{*}\left[1+\left(E-E_{c}\right) / E_{g}\right]$ into Eq. (3b). The plots $E(k)$ predicted by Eq. (4) for an exemplary quantum well with two confined states at $E_{1} \cong 0.15 \mathrm{eV}$ and $E_{2} \cong 0.413 \mathrm{eV}$ are shown in Fig. 1. The deviation from the dispersion relation predicted by the 8-band kp model for the lower state is even smaller than that for Eq. (1). Moreover, there is no evidence which approximation is better because the 8-band $\mathrm{kp}$ model is also an approximation which 
Fig. 1 In-plane dispersion relation calculated for Eq. (1) (solid black lines), or Eq. (3) (dashed red lines) for $4.8 \mathrm{~nm}$ wide InGaAs/AlInAs quantum well (lattice matched to InP) compared to the 8-band $\mathrm{kp}$ model calculations of Faist (2013) (dotted blue lines) and the 14-band kp model (dash-dotted green lines) of Ekenberg (1989). (Color figure online)

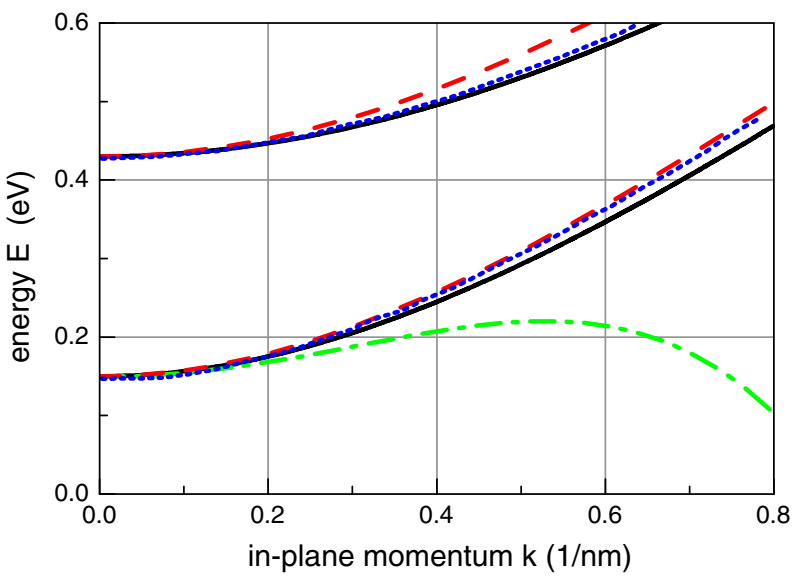

introduces inaccuracy at high in-plane momentum. This is evident when compared to more exact results predicted by a 14-band kp model (Ekenberg 1989) (see Fig. 1).

The subscript 'aw' in Eq. (3) abbreviates 'active wells', so $z_{\text {aw }}$ points at the wells where a major optical transition takes place. Fixing $z$ at $z_{\text {aw }}$ in Eq. (3b) makes longitudinal energy loose its spatial dependence. Then $E_{z}$ becomes a real number and Eq. (3a) can be rewritten in the form

$$
\frac{-\hbar^{2}}{2} \frac{d}{d z} \frac{1}{m\left(E_{z}, z\right)} \frac{d f}{d z}+E_{c}(z) f=E_{z} f,
$$

which has the energy-independent TB counterpart

$$
\left[\begin{array}{cc}
E_{c}(z) & \frac{\hbar^{2}}{2 m^{*} \sqrt{\gamma}} \frac{d}{d z} \\
-\frac{\hbar^{2}}{2 m^{*} \sqrt{\gamma}} \frac{d}{d z} & E_{v}(z)
\end{array}\right]\left[\begin{array}{l}
f \\
g
\end{array}\right]=E_{z}\left[\begin{array}{l}
f \\
g
\end{array}\right] .
$$

Equation (6) provides the orthonormal solutions $(f, g)$ which can be used in the calculations of the momentum matrix elements (Leavitt 1991; Sirtori et al. 1994).

\section{Nonequilibrium Green's function (NEGF) implementation}

Equation (3) defines a $k$-dependent Hamiltonian which can be used in Nonequilibrium Green's Function (NEGF) method. In the real space implementation, the conduction band Green's functions (GFs) $G^{R}, G^{<}$are the four-parameter functions of positions $z, z^{\prime}$, energy $E$, and in-plane momentum modulus $k$. For discretized Hamiltonians, useful in numerical simulations and offering a compact form of integral equations, the retarded $\mathrm{GF}, \mathbf{G}^{\mathbf{R}}(E, k)$, is the solution of the Dyson equation (Kubis et al. 2009)

$$
\left(E \mathbf{I}-\mathbf{H}-\boldsymbol{\Sigma}^{\mathbf{R}}\right) \mathbf{G}^{\mathbf{R}}(E, k)=\mathbf{I},
$$

where the self-energy term $\Sigma^{\mathbf{R}}$ comprises scatterings and couplings to the leads. The valence component of the retarded GF is given by Kolek (2015) 


$$
\mathbf{G}_{v \mathbf{c}}^{\mathbf{R}}(\mathbf{E}, \mathbf{k})=\left(\mathbf{E}_{\mathbf{z}}-\mathbf{E}_{v}\right)^{-\mathbf{1}} \mathbf{H}_{\mathbf{c} v}^{\mathbf{t}} \mathbf{G}^{\mathbf{R}}(\mathbf{E}, \mathbf{k}), \mathbf{G}_{v \mathbf{c}}^{\mathbf{R}}(\mathbf{E}, \mathbf{k})=\left(\mathbf{E}_{\mathbf{z}}-\mathbf{E}_{v}\right)^{-\mathbf{1}}\left[\mathbf{I}+\mathbf{H}_{\mathbf{c} v}^{\mathbf{t}} \mathbf{G}_{\mathbf{c} v}^{\mathbf{R}}(\mathbf{E}, \mathbf{k})\right],
$$

where $\mathbf{H}_{\mathbf{c} v}$ is the discretized differential operator

$$
\frac{\hbar^{2}}{2 m^{*} \sqrt{\gamma}} \frac{d}{d z} \rightarrow \mathbf{H}_{\mathbf{c} v} \text { and } \mathrm{E}_{\mathrm{c}}(\mathrm{z})-\mathrm{E}_{\mathrm{g}}(\mathrm{z})=\mathrm{E}_{v}(\mathrm{z}) \rightarrow \mathbf{E}_{v} .
$$

Lesser Green's functions can be obtained from the Keldysh equation

$$
\left[\begin{array}{ll}
\mathbf{G}^{<} & \mathbf{G}_{\mathbf{c} v}^{<} \\
\mathbf{G}_{v \mathbf{c}}^{<} & \mathbf{G}_{v}^{<}
\end{array}\right]=\left[\begin{array}{ll}
\mathbf{G}^{\mathbf{R}} \boldsymbol{\Sigma}^{<} \mathbf{G}^{\mathbf{R}^{*}} & \mathbf{G}^{\mathbf{R}} \boldsymbol{\Sigma}^{<} \mathbf{G}_{v \mathbf{c}}^{\mathbf{R}^{*}} \\
\mathbf{G}_{v \mathbf{c}}^{\mathbf{R}} \boldsymbol{\Sigma}^{<} \mathbf{G}^{\mathbf{R}^{*}} & \mathbf{G}_{v \mathbf{c}}^{\mathbf{R}} \boldsymbol{\Sigma}^{<} \mathbf{G}_{v \mathbf{c}}^{\mathbf{R}^{*}}
\end{array}\right],
$$

where $\boldsymbol{\Sigma}^{<}$is the conduction band lesser self-energy. Equations (7-9) together with the Poisson equation should be iterated. In this iteration, the GFs valence components $\mathbf{G}_{v}^{\mathbf{R}}, \mathbf{G}_{v}^{<}$ should be included as they contribute to the density of states (DOS) and the density of electrons (DOE)

$$
\begin{gathered}
N(E, k, z)=-\frac{1}{\pi a} \operatorname{Im}\left[\mathrm{G}^{\mathrm{R}}(\mathrm{E}, \mathrm{k}, \mathrm{z}, \mathrm{z})+\mathrm{G}_{v}^{\mathrm{R}}(\mathrm{E}, \mathrm{k}, \mathrm{z}, \mathrm{z})\right], \\
n(E, k, z)=-\frac{i}{2 \pi a}\left[G^{<}(E, k, z, z)+G_{v}^{<}(E, k, z, z)\right],
\end{gathered}
$$

and thus influence the GFs through the Poisson equation. Once the self-consistency is achieved, the optical absorption can be evaluated making use of the approach developed by Wacker (2002) applied to the TB Hamiltonian (Kolek 2015).

\section{Quantum cascade laser}

The model described in Sect. 2 is especially useful for a quantum cascade laser (QCL) - a device consisting of tens of layers with carefully tuned width what calls for a very dense discretization mesh in the simulations. This generates huge matrices and makes multiband modeling hardly possible. On the contrary, the one-band approach keeps the matrices in reasonable size and enables the use of the NEGF method, which treats quantum tunneling and scattering on equal footing (Kubis et al. 2009) what is necessary for this type of devices.

The model of Sect. 2 and its implementation of Sect. 3 were used to calculate electronic transport and optical gain in QCL emitting at $\cong 5 \mu \mathrm{m}(h v \cong 0.25 \mathrm{eV})$, designed by Evans et al. (2007). The equations of the NEGF formalism were solved for one QCL module with the contact self-energies which mimic periodic boundary conditions (Haldas et al. 2011; Kolek et al. 2012). Other self-energies included into formalism represent electron-phonon (LO, LA), interface roughness, alloy disorder and ionized impurity scatterings. Carrier-carrier interaction was included as the mean-field through the Poisson equation. The discretization mesh spacing was $0.6 \mathrm{~nm}$. In Fig. 2, which shows exemplary results, the focus is paid on the $k$-resolved quantities which demonstrate in-plane nonparabolicity inherently contained in the model. Preserving this feature is crucial for realistic modeling of intersubband gain. As discussed by Faist et al. (1996), in QCLs the optical gain can emerge due to the local (in $k$-space) population inversion which is not 


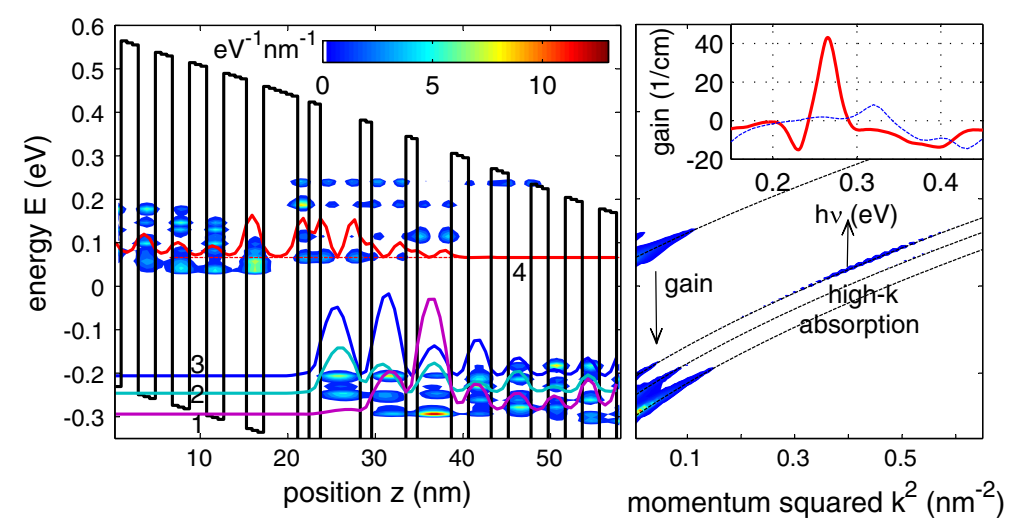

Fig. 2 (left) Spectral function at $k=0$, and (right) the subband occupation in active wells of QCL design of Evans et al. (2007). Population inversion is observed only at low- $k$ momenta, where the occupation of the upper laser subband 4 exceeds the occupation of the lower laser subband $3.4 \rightarrow 3$ transitions at high$k$ values, where the occupation is normal, do not destroy gain because they absorb photons with lower energy: $h v_{\text {high-k }}<h v_{\text {low-k}}$. These transitions burn a hole in front of the gain peak in the gain spectrum as shown in the inset. Blue (dashed) line in the inset shows gain spectrum (multiplied $\times 10$ ) calculated for the one-band model which uses constant effective masses in well and barrier materials (calculations were made for the same value of electric field). (Color figure online)

destroyed by the absorption at higher $k$-values due to the in-plane nonparabolicity. Indeed, our simulations show that substantial gain emerges (see Fig. 2 inset) (due to $4 \rightarrow 3$ transitions) at the energy $h v \cong 0.26 \mathrm{eV}$ that agrees very well with the experimental lasing wavelength. This is in sharp contrast with purely one-band model which predicts both wrong lasing energy and almost no gain: for this case, the gain peak of merely $0.8 \mathrm{~cm}^{-1}$ occurs at $h v=0.32 \mathrm{eV}$ ! Results of one-band simulations are also shown in Fig. 2 for the comparison.

When gain emerges due to local population inversion (which is our case) the most important is an accurate modeling of the in-plane dispersion at low and medium $k$-values, where intersubband transitions occur at nearly the same values of the photon energy and thus contribute to the gain spectrum. The photons emitted at remote $k$ 's have the energies well apart from the energy emitted in the transitions at $k \cong 0$ and so hardly influence the value of the gain peak. Then, going beyond the accuracy of 8-band description, which one may concern looking at the deviations in Fig. 1, seems to be both hard and impractical, at least in the case of QCLs. The model defined by Eq. (3) preserves the accuracy of 8-band model at low $k$-values which, as shown, is sufficient enough to provide reliable results.

The gain in Fig. 2 inset was calculated as the negative absorption coefficient averaged over one laser period. Both the conduction and the valence components of GFs were involved in these calculations. The amount of the valence component can be estimated, e.g., from the contribution to the total electron density. The plots in Fig. 3 allow to estimate that it contributes to $\cong 5$ percent of the total charge. This number illustrates how much charge one looses if does not care about the valence component what is usually done in one-band calculations. Worth to note is that through the Poisson equation this component affects not only DOE but all other quantities including the intersubband gain.

In summary, a one-band effective mass model was proposed which allows for $k$-resolved calculations which preserve correct nonparabolicity both in transport and in-plane directions and allows for rigorous calculations of intersubband absorption which takes these (nonparabolicities) into-account. 


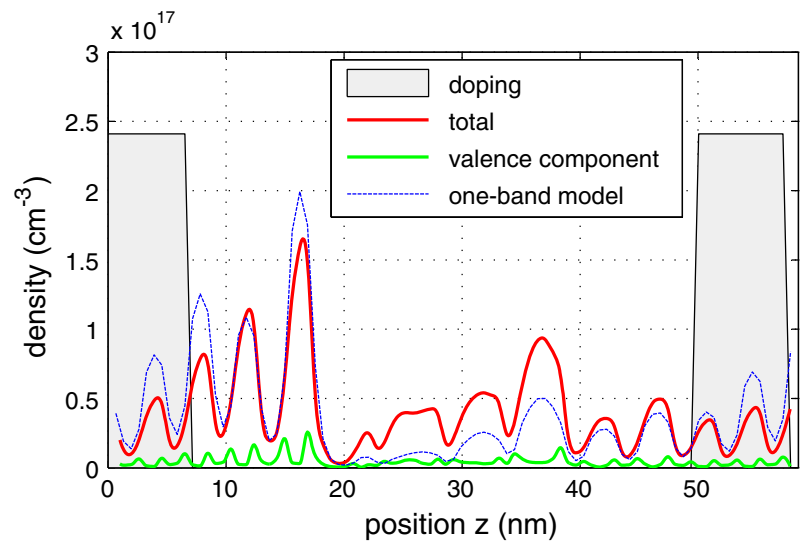

Fig. 3 Total density of electrons (red line) in one module of QCL. The valence contribution is marked with green line. Shaded area shows doping. Dashed (blue) line shows electron density calculated for purely oneband model (for the same electric field). (Color figure online)

Acknowledgments The work was supported by the Polish research supporting agencies: the National Science Center (NCN), under the project SONATA 2013/09/D/ST7/03966 and the National Center for Research and Development (NCBR) under the grant PBS 1/B3/2/2012 (EDEN).

Open Access This article is distributed under the terms of the Creative Commons Attribution 4.0 International License (http://creativecommons.org/licenses/by/4.0/), which permits unrestricted use, distribution, and reproduction in any medium, provided you give appropriate credit to the original author(s) and the source, provide a link to the Creative Commons license, and indicate if changes were made.

\section{References}

Ekenberg, U.: Nonparabolicity effects in a quantum well: Sublevel shift, parallel mass, and Landau levels. Phys. Rev. B 40, 7714-7726 (1989)

Evans, A., Darvish, S.R., Slivken, S., Nguyen, J., Bai, Y., Razeghi, M.: Buried heterostructure quantum cascade lasers with high continuous-wave wall plug efficiency. Appl. Phys. Lett. 91, 071101 (2007)

Faist, J., Capasso, F., Sirtori, C., Sivco, D.L., Hutchinson, A.L., Hybertsen, M.S., Cho, A.Y.: Quantum cascade lasers without intersubband population inversion. Phys. Rev. Lett. 76, 411-414 (1996)

Faist, J.: Quantum Cascade Lasers. Oxford University Press, Oxford (2013)

Haldas, G., Kolek, A., Tralle, I.: Modeling of mid-infrared quantum cascade laser by means of nonequilibrium Green's functions. IEEE J. Quantum Electron. 47, 878-885 (2011)

Kolek, A., Haldas, G., Bugajski, M.: Nonthermal carrier distributions in the subbands of 2-phonon resonance mid-infrared quantum cascade laser. Appl. Phys. Lett. 101, 061110 (2012)

Kolek, A.: Nonequilibrium Green's function formulation of intersubband absorption for nonparabolic single-band effective mass Hamiltonian. Appl. Phys. Lett. 106, 181102 (2015)

Kubis, T., Yeh, C., Vogl, P.: Theory of nonequilibrium quantum transport and energy dissipation in terahertz quantum cascade lasers. Phys. Rev. B 79, 195323 (2009)

Leavitt, R.P.: Empirical two-band model for quantum wells and superlattices in an electric field. Phys. Rev. B 44, 11270-11280 (1991)

Sirtori, C., Capasso, F., Faist, J., Scandolo, S.: Nonparabolicity and a sum rule associated with bound-tobound and bound-to-continuum intersubband transitions in quantum wells. Phys. Rev. B. 50, 8663-8674 (1994)

Wacker, A.: Gain in quantum cascade lasers and superlattices: a quantum transport theory. Phys. Rev. B. 66, 085326 (2002) 